THEORY OF COMPUTING, Volume 5 (2009), pp. 125-134

www.theoryofcomputing.org

\title{
Hard Metrics From Cayley Graphs Of Abelian Groups
}

\author{
Ilan Newman $\quad$ Yuri Rabinovich*
}

Received: April 29, 2008; published: July 3, 2009.

\begin{abstract}
Hard metrics are the class of extremal metrics with respect to embedding into Euclidean spaces; they incur $\Omega(\log n)$ multiplicative distortion, which is as large as it can possibly get for any metric space of size $n$. Besides being very interesting objects akin to expanders and good error-correcting codes, and having a rich structure, such metrics are important for obtaining lower bounds in combinatorial optimization, e. g., on the value of MinCut/MaxFlow ratio for multicommodity flows.

For more than a decade, a single family of hard metrics was known (Linial, London, Rabinovich (Combinatorica 1995) and Aumann, Rabani (SICOMP 1998)). Recently, a different family was found by Khot and Naor (FOCS 2005).

In this paper we present a general method of constructing hard metrics. Our results extend to embeddings into negative type metric spaces and into $\ell_{1}$.
\end{abstract}

ACM Classification: F.2.2, G.2.2

AMS Classification: $11 \mathrm{~J} 83$, 52C45, 05C25

Key words and phrases: Metric Embedding, Hard Metrics

\section{Introduction}

A famous theorem of Bourgain [4] states that every finite metric space $V=(X, d)$ of size $n=|X|$ can be embedded into a Euclidean space with multiplicative distortion at most $O(\log n)$. We call a metric space $V$ hard with respect to $\ell_{2}$ if any embedding of $V$ into a Euclidean space (of any dimension) has a multiplicative distortion $\Omega(\log n)$. Similarly, we call a metric space $V$ hard with respect to $\mathcal{M}$, where $\mathcal{M}$ is a class of metric spaces (e.g., $\mathcal{M}=\ell_{p}$ or NEG), if any embedding of $V$ into $\mathcal{M}$ has multiplicative

* Supported in part by a grant ISF-247-020-10.5 


\section{NEWMAN, AND Y. RABINOVICH}

distortion $\Omega(\log n)$. When we use the term hard without specifying $\mathcal{M}$, we always mean with respect to $\mathcal{M}=\ell_{2}$.

When studying a special class of metric spaces, perhaps the most natural first question is whether this class contains hard metrics with respect to $\ell_{2}$. Many fundamental results in the modern theory of finite metric spaces may be viewed as a negative answer to this question for some special important class of metrics. E. g., Arora et al. [2] (improving on Chawla et al. [5]) show this for Negative Type metrics, Rao [14] for planar metrics, and Gupta et al. [7] for doubling metrics. For a long time (since Linial, London and Rabinovich [11] and Rabani and Aumann [3]), the only known family of hard metrics was, essentially, the shortest-path metric of constant-degree expander graphs. It was even suggested that in some vague sense these are essentially the only hard metrics. Recently, however, Khot and Naor [10] constructed a different family of hard metrics by considering certain quotient spaces of $\mathbb{Z}_{2}^{n}$ equipped with the Hamming distance.

The starting point of the current research was a plausible conjecture that a circular metric cannot be hard, where by circular we mean a metric on the underlying space $\mathbb{Z}_{n}$, such that $d(a, b)$ depends solely on $((a-b) \bmod n)$. Rather surprisingly, the conjecture turns out to be false, and, moreover, it fails not only for $\mathbb{Z}_{n}$, but for any Abelian group $H$. More precisely, it is always possible to choose a set $A$ of generators for $H$, so that the shortest-path metric of the corresponding Cayley graph $G(H, A)$ is hard with respect to $\ell_{2}, \ell_{1}$ and NEG. In the special case of $\mathbb{Z}_{2}^{n}$, good sets of generators are closely related to error-correcting codes of constant rate and linear distance.

Our construction is both simple to describe and easy to analyze. It differs from that of [11,3], as the degree of such Cayley graphs is necessarily not bounded. Moreover, the construction of [10], despite very different description and analysis, can be shown to produce the same metric space as does our construction in the special case of $\mathbb{Z}_{2}^{n}$.

Note: Although in what follows we restrict the discussion to Euclidean spaces, the same method can be used to show the hardness of the metrics that we construct also with respect to the much richer class NEG of "negative type metrics" and consequently to $\ell_{1}$.

\section{Definitions}

Let $(X, d)$ be a metric space which one wants to embed into another metric space $A=(H, v)$. The multiplicative distortion, or simply the distortion of embedding $(X, d)$ into $A$ is defined as

$$
c_{A}(d)=\operatorname{dist}(d \hookrightarrow A)=\min _{\phi: X \rightarrow H} \max _{x, y \in X} \frac{v(\phi(x), \phi(y))}{d(x, y)} \cdot \max _{x, y \in X} \frac{d(x, y)}{v(\phi(x), \phi(y))} .
$$

We use the terms Euclidean metrics and $\ell_{2}$-metrics interchangeably.

We say that a metric space $V=(X, d)$ is of negative type if there is a map $f$ from $X$ to an Euclidean space such that for all $x, y \in X$ we have $d(x, y)=\|f(x)-f(y)\|_{2}^{2}$. (So all triangles spanned by the image of $X$ are acute.) NEG denotes the class of negative type metrics.

It is well known that $\ell_{1}$ metrics are of negative type (cf. [6], Part 1, Chapter 6.1 for a comprehensive discussion and historical notes). 


\section{Abelian Groups}

Let $G=(V, E)$ be a $d$-regular connected graph on $n$ vertices, and let $\mu_{G}$ be its shortest-path metric. For $\Delta: V(G) \times V(G) \rightarrow \mathbb{R}^{+}$, consider the following projective quadratic form, often called a Poincaré form:

$$
F(\Delta)=\frac{\sum_{(i, j) \in E(G)} \Delta^{2}(i, j)}{\sum_{i<j \in V(G)} \Delta^{2}(i, j)}
$$

To obtain distortion lower bounds on $\mu_{G}$, we use the standard (dual) method of comparing Poincaré forms (see, e. g., $[11,13])$. Our first step is to get a general lower bound on distortion of embedding $\mu_{G}$ into an Euclidean space.

By the definition above,

$$
F\left(\mu_{G}\right)=\frac{|E|}{\left(\begin{array}{l}
n \\
2
\end{array}\right) \operatorname{avg}\left(\mu_{G}^{2}\right)},
$$

where $\operatorname{avg}\left(\mu_{G}^{2}\right)$ is the average value of $\mu_{G}^{2}(i, j)$ over all pairs $(i, j)$ of distinct vertices of $G$.

Consider now a Euclidean metric on $V(G), \delta \in \ell_{2}$, namely, a metric of the form

$$
\delta(i, j)=\left\|x^{i}-x^{j}\right\|_{2}, \quad\left\{x^{i}\right\}_{i \in V(G)} \subset \mathbb{R}^{m} .
$$

If $F(\delta)$ is much larger than $F\left(\mu_{G}\right)$ for every such $\delta$, one immediately concludes that any such $\delta$ must significantly distort $\mu_{G}$. Formally,

\section{Proposition 3.1.}

$$
\operatorname{dist}^{2}\left(\mu_{G} \hookrightarrow \ell_{2}\right) \geq \min _{\delta \in \ell_{2}} F(\delta) / F\left(\mu_{G}\right) .
$$

By a standard argument (see e.g., [13], Sect. 15.5), the minimum of $F(\delta)$ over all such $\delta$ is precisely $\gamma_{G} / n$, where $\gamma_{G}$ is the spectral gap of $G$, that is, $\left(d-\lambda_{G}\right)$ where $\lambda_{G}$ is the second largest eigenvalue of the adjacency matrix of $G$. Thus, Proposition 3.1 implies,

\section{Proposition 3.2.}

$$
\operatorname{dist}^{2}\left(\mu_{G} \hookrightarrow \ell_{2}\right) \geq \frac{n-1}{n} \cdot \frac{\gamma_{G}}{d} \cdot \operatorname{avg}\left(\mu_{G}^{2}\right)
$$

In particular,

Corollary 3.3. Let $\left\{G_{n}\right\}$ be a family of regular graphs; assume $G_{n}$ has $n$ vertices and degree $d_{n}$. Suppose the normalized spectral gaps $\gamma_{G_{n}} / d_{n}$ are bounded away from zero and $\operatorname{avg}\left(\mu_{G_{n}}^{2}\right)=\Omega\left(\log ^{2} n\right)$. Then the distance metric for this family of graphs is hard.

In what follows we shall deal with families of graphs for which $\operatorname{avg}\left(\mu_{G}^{2}\right)=\Omega\left(\operatorname{Diam}(G)^{2}\right)$. We note that, in particular, any vertex-transitive graph has this property. A graph is vertex-transitive if all of its vertices are equivalent under automorphisms.

Proposition 3.4. If $G$ is a vertex-transitive graph then $\operatorname{avg}\left(\mu_{G}^{2}\right) \geq \operatorname{Diam}(G)^{2} / 8$.

Proof. Indeed, let $r$ be the smallest radius such that the corresponding $r$-ball in $\mu_{G}$ contains more than $n / 2$ vertices. Clearly, $\operatorname{avg}\left(\mu_{G}^{2}\right) \geq r^{2} / 2$, while $\operatorname{Diam}(G) \leq 2 r$. 


\section{NEWMAN, AND Y. RABINOVICH}

Therefore, for vertex-transitive graphs, it suffices to ensure a constant normalized spectral gap and an $\Omega(\log n)$ lower bound on the diameter.

Turning to Cayley graphs, it is well known that for (some) classes of non-Abelian groups, there exist Cayley graphs with a bounded number of generators, and a constant spectral gap (see, e.g., [8], the section on Cayley expander graphs). Since the constant number of generators guarantees that the diameter is $\Omega(\log n)$, this yields a graph as required in Corollary 3.3. (This is precisely the construction used in [11,3]). For Abelian groups such construction is impossible, since in order to ensure a constant normalized gap $\gamma_{G} / d$, the number of generators must be at least $\Omega(\log n)$ (see, e. g., [8]). This might seem to be a problem, since, at least for general groups, that many generators may well cause the diameter to be $O(\log n / \log \log n)=o(\log n)$. For Abelian groups, however, this does not happen! While the following simple fact is well known (see, e.g., [8], proof of Prop. 11.5), it has apparently been overlooked in the context of hard metrics.

Let $h(p)=-p \log _{2} p-(1-p) \log _{2}(1-p)$ be the binary entropy function. For an Abelian group $H$, a set $A \subseteq H$ is called symmetric if $A=-A$ (we use the additive notation for the Abelian group operation).

Proposition 3.5. Let $H$ be an Abelian group of order $n$, and let $A \subset H$ be a symmetric set of generators of size $d=c_{0} \log _{2} n$. Then, for any constant $c_{1}$ such that $\left(c_{0}+c_{1}\right) \cdot h\left(c_{1} /\left(c_{0}+c_{1}\right)\right)<1$, the diameter of the corresponding Cayley graph $G(H, A)$ is $\geq c_{1} \log _{2} n$ for a large enough $n$.

The proposition follows from the observation that the number of distinct endpoints of paths of length $l$ in $G$ starting at any (fixed) vertex is at most $\left(\begin{array}{c}d+l \\ l\end{array}\right)$, since due to commutativity of $G$ it is at most the number of partitions of a set of $l$ identical elements to $d$ (distinct) parts. Therefore, the number of points reachable by a path of length $\leq c_{1} \log _{2} n$ from a fixed vertex is at most

$$
\begin{gathered}
\sum_{l=0}^{c_{1} \log _{2} n}\left(\begin{array}{c}
c_{0} \log _{2} n+l \\
l
\end{array}\right) \leq 2^{h\left(\frac{c_{1}}{c_{0}+c_{1}}\right) \cdot\left(c_{0}+c_{1}\right) \cdot \log _{2} n}= \\
n^{\left(c_{0}+c_{1}\right) \cdot h\left(\frac{c_{1}}{c_{0}+c_{1}}\right)}<n .
\end{gathered}
$$

Thus, as long as the number of generators is $O(\log n)$, our only concern is getting a constant normalized spectral gap $\gamma_{G} / d$. This is summed up in the following theorem.

Theorem 3.6. Let us fix an arbitrary constant $c_{0}>0$. Let $H$ be an Abelian group of order $n$, let $A \subset H$ be a symmetric set of generators of size $d=c_{0} \log _{2} n$ and let $G(H, A)$ be the corresponding Cayley graph. If the normalized spectral gap $\gamma_{G} /|A|=\Omega(1)$, then $\mu_{G}$ is a hard metric.

It is well known that random choice achieves constant spectral gap (see, e. g., [1], in particular the section on Abelian groups):

Proposition 3.7. Let $H$ be an Abelian group of order $n$, and let $A \subset H$ be a random symmetric set of generators of size $d=c_{0} \log _{2} n$ for a suitable universal constant $c_{0}(100$ would certainly suffice $)$. Then, the corresponding Cayley graph $G(H, A)$ almost surely has a normalized spectral gap $\geq 0.5$.

For an efficient deterministic construction of such sets $A$ (for any group, not only Abelian groups) see [15, Sec. 5]. Combining Theorem 3.6 and Proposition 3.7, we arrive at the main result of this section: 
Theorem 3.8. Let $G=G(H, A)$ be a Cayley graph obtained by taking a random symmetric set of generators $A \subset H$ of size $d=c_{0} \log _{2}|H|$ for a suitable universal constant $c_{0}$. Then, the shortest-path metric of $G$ is almost surely a hard metric.

Remark: Using the linear projective form

$$
F_{1}(\Delta)=\frac{\sum_{(i, j) \in E(G)} \Delta(i, j)}{\sum_{i<j \in V(G)} \Delta(i, j)}
$$

instead of $F(\Delta)$, implies the following for negative type metrics,

$$
\operatorname{dist}\left(\mu_{G} \hookrightarrow \mathrm{NEG}\right) \geq \min _{\delta \in \mathrm{NEG}} F_{1}(\delta) / F_{1}\left(\mu_{G}\right) .
$$

Arguing along the same lines as for Euclidean metrics (and recalling that a metric in NEG is a square of an $\ell_{2}$ metric), it can be seen that the metrics of Theorem 3.8 are hard with respect to NEG as well.

\section{When the Group is $\mathbb{Z}_{2}^{n}$}

In this case the group is just an $n$-dimensional vector space over $\mathbb{Z}_{2}$. Any set of generators (vectors) $A$ is automatically symmetric. Following the requirements of Corollary 3.3, we have to ensure two conditions: a constant normalized spectral gap and $\Omega(n)$ diameter.

The construction is based on good linear codes. Let $\mathcal{C} \subset \mathbb{Z}_{2}^{m}$ be a linear code (subspace) of dimension $n$. The weight $w(v)$ of a vector $v$ is the number of nonzero entries of $v$. The distance $D(\mathcal{C})$ of $\mathcal{C}$ is the minimum weight of nonzero vectors in $\mathcal{C}$. $\mathcal{C}$ is said to be of linear distance if $D(\mathcal{C})=\Omega(m)$. In addition, if $n=\Omega(m)$ the code is said to have a constant rate.

Let $M$ be an $n \times m$ matrix whose rows form a basis for $\mathcal{C}$ (such an $M$ is called the generator matrix of $\mathcal{C})$.

Proposition 4.1. Let $\mathcal{C} \subset \mathbb{Z}_{2}^{m}$ be a linear code. Let $M$ be the corresponding $n \times m$ matrix and $A$ the set of columns of $M$ as above. Then the spectral gap of the Cayley graph $G=G\left(\mathbb{Z}_{2}^{n}, A\right)$ is $\gamma_{G}=2 D(\mathcal{C})$.

It follows that the normalized spectral gap $\gamma_{G} / m$ is bounded away from zero if and only if $\mathcal{C}$ is a code of linear distance.

The proposition is folklore (see e. g. [1], proof of Proposition 2). Here is a sketch of the proof.

Proof. The characters $\left\{\chi_{u}\right\}$ of $\mathbb{Z}_{2}^{n}$, indexed by the group elements $u \in \mathbb{Z}_{2}^{n}$, are of the form

$$
\chi_{u}(x)=(-1)^{\langle u, x\rangle},
$$

where the inner product is mod 2. Let $A \subset \mathbb{Z}_{2}^{n},|A|=m$, be a set of generators (vectors), and let $M_{A}$ be an $n \times m$ matrix over $\mathbb{Z}_{2}$ whose columns are the vectors of $A$. Keeping in mind that the eigenvectors of $G\left(\mathbb{Z}_{2}^{n}, A\right)$ are the characters, we conclude that the second largest eigenvalue $\lambda_{G}$ of $G\left(\mathbb{Z}_{2}^{n}, A\right)$ is

$$
\lambda_{G}=\max _{u \in \mathbb{Z}_{2}^{n} \backslash\{0\}} \sum_{a \in A}(-1)^{\langle u, a\rangle}=\max _{u \in \mathbb{Z}_{2}^{n} \backslash\{0\}}\left\{m-2 w\left(u^{T} M_{A}\right)\right\} .
$$




\section{NEWMAN, AND Y. RABINOVICH}

Let $\mathcal{C} \subseteq \mathbb{Z}_{2}^{n}$ be a linear code generated by $M_{A}$, that is, all linear combinations of the rows of $M_{A}$. Then $\mathcal{C}=\left\{u^{T} M_{A}\right\}_{u \in \mathbb{Z}_{2}^{n}} \subset \mathbb{Z}_{2}^{m}$ and hence $\lambda_{G}=m-2 D(\mathcal{C})$. Since $\gamma_{G}=m-\lambda_{G}$, it follows that $\gamma_{G}=2 D(\mathcal{C})$. Therefore, $\gamma_{G}=\Omega(m)$ if and only $\mathcal{C}$ is a linear code of linear distance.

It remains to ensure that the diameter of $G\left(\mathbb{Z}_{2}^{n}, A\right)$ is $\Omega(n)$. By Proposition 3.5, this condition will necessarily hold provided $m=O(n)$, that is, if $\mathrm{C}$ is of constant rate. We thus proved the following.

Theorem 4.2. Let $\mathrm{C}$ be a linear code of constant rate and linear distance, and $\operatorname{dim}(\mathrm{C})=n$. Let $M$ be an $n \times m$ matrix whose rows form a basis for $\mathrm{C}$, and let $A \subset \mathbb{Z}_{2}^{n}$ be the set of columns of $M$. Then the metric of $G\left(\mathbb{Z}_{2}^{n}, A\right)$ is hard.

Linear codes of constant rate and linear distance have received considerable attention. Their existence has been established by numerous randomized and deterministic efficient constructions, with the first explicit construction due to Justesen [9] (cf. [12]).

We conclude this section with a comparison of the construction of hard metrics due to Khot and Naor [10] and our construction. Let $\mathcal{C} \subset \mathbb{Z}_{2}^{m}$ be a linear code of constant rate and linear distance, of dimension $n$. Let $\mathcal{C}^{\perp}$ be the dual code, i. e., $\mathcal{C}^{\perp}=\{u \mid M u=0\}$ where $M$ is the generator matrix of $\mathcal{C}$. Define an equivalence relation on $\mathbb{Z}_{2}^{m}$ by $x \equiv y$ if $(x-y) \in \mathrm{C}^{\perp}$. Now, let $X$ be a quotient metric space of $\mathbb{Z}_{2}^{m}$ equipped with the Hamming metric, with respect to $\equiv$. That is, the distance between two points $a$ and $b$ in $X$ is the Hamming distance between the two corresponding cosets $A, B \subset \mathbb{Z}_{2}^{m}$. Khot and Naor show that $X$ with the induced metric is hard.

Proposition 4.3. The above construction is isometric to the construction described in Theorem 4.2.

Proof. Let $M$ be a matrix as in Theorem 4.2. Then $X$ can be viewed as the image of $\mathbb{Z}_{2}^{m}$ under the linear mapping $\phi: \mathbb{Z}_{2}^{m} \rightarrow \mathbb{Z}_{2}^{n}, \phi(x)=M x$. Define the edges of $X$ as the images of Hamming edges of $\mathbb{Z}_{2}^{m}$ under $\phi$. Clearly, the quotient metric of $X$ is precisely the shortest-path metric of the resulting graph. The images of the Hamming edges are, however, precisely the column vectors of $M$, and the isometry follows.

\section{Additional Remarks}

The constructions of Cayley graphs with hard shortest-path metric as described in Theorem 3.8 and Theorem 4.2, yield graphs of degree logarithmic in the number of vertices. It is natural to ask whether this must hold for all Cayley graphs of Abelian groups that induce a hard metric. Here we partially answer this question and show that the degree can be anything between $\Omega(\log n)$ and $O\left(n^{1-\varepsilon}\right)$ for any fixed $1>\varepsilon>0$.

We start with the following simple fact:

Proposition 5.1. Let $H$ be an Abelian group, and let $m<|H|$ be a natural number. Then, there exists a symmetric set $B \subseteq H$ of size $\Theta(m)$ such that for every natural $r$ the size of $r B=\left\{\sum_{i=1}^{r} b_{i} \mid b_{i} \in B\right\}$ is at most $r \cdot|B|$. 
Proof. Any finite Abelian group is a direct product of cyclic groups. Let $H=C_{1} \times C_{2} \times \ldots \times C_{t}$, and assume that $s_{j}=\left|C_{1}\right| \cdot\left|C_{2}\right| \cdot \ldots \cdot\left|C_{j}\right|<m$, while $s_{j+1}=\left|C_{1}\right| \cdot\left|C_{2}\right| \cdot \ldots \cdot\left|C_{j}\right| \cdot\left|C_{j+1}\right| \geq m$. Let $a$ be a generator of $C_{j+1}$. Define $K_{j+1}=\{i \cdot a \mid i \in[-k, k] \subset \mathbb{N}\}$ where $k$ is the smallest natural number such that $s_{j} \cdot(2 k+1) \geq m$. Finally, define $B=C_{1} \times C_{2} \times \ldots \times C_{j} \times K_{j+1} \times\{0\} \times\{0\} \times \ldots$ It is easy to verify that $B$ has the required properties.

Theorem 5.2. Let $H$ be an Abelian group of order $n$, and let $1>\varepsilon>0$ be fixed. Then there exists $a$ symmetric set of generators, $A$, of size $\Theta\left(n^{1-\varepsilon}\right)$, such that the metric $\mu_{G}$ of the Cayley graph $G=G(H, A)$ is hard.

Proof. Let $G^{\prime}=G^{\prime}(H, A)$ be a Cayley graph as in Theorem 3.8 (or Theorem 4.2), with $|A|=c_{0} \log _{2} n$. Assume for simplicity that $A$ is augmented by $\{0\}$. Let $B \subseteq H$ be as in Proposition 5.1 with $m=n^{1-\varepsilon}$. We claim that the Cayley graph $G=G(H, A \cup B)$ has the desired properties. To see that, we employ the Poincaré form $F^{\prime}(\Delta)$ similar to $F(\Delta)$ of (3.1), where in the numerator we use the edges of $G^{\prime}$ instead of the edges of $G$. Arguing as in Proposition 3.2, we conclude that

$$
\operatorname{dist}^{2}\left(\mu_{G} \hookrightarrow \ell_{2}\right) \geq \frac{n-1}{n} \cdot \frac{\gamma_{G^{\prime}}}{d^{\prime}} \cdot \operatorname{avg}\left(\mu_{G}^{2}\right)
$$

We already know that the normalized spectral gap of $G^{\prime}$ is constant. Thus, it will suffice to show that the diameter of $G$ is logarithmic. A closer examination of the proof of Proposition 3.5 reveals that the number of distinct endpoints of paths of length $\leq c_{\varepsilon / 2} \log _{2} n$ starting at a fixed vertex in $G^{\prime}$, is at most $n^{\varepsilon / 2+o(1)}$, provided that $\left(c_{0}+c_{\varepsilon / 2}\right) \cdot h\left(c_{\varepsilon / 2} /\left(c_{0}+c_{\varepsilon / 2}\right)\right) \leq \varepsilon / 2$. Therefore, the number of points reachable by a path of length at most $r=c_{\varepsilon / 2} \log _{2} n$ in $G$ is

$$
|r \cdot\{A \cup B\}| \leq|r A| \cdot|r B| \leq|r A| \cdot r|B| \leq n^{\varepsilon / 2} \cdot n^{1-\varepsilon} \cdot \Theta\left(n^{o(1)}\right)<n .
$$

(At most $r$ rather than exactly $r$ since both $A$ and $B$ contain 0 .) Thus, the diameter of $G$ is at least $c_{\varepsilon / 2} \log _{2} n$. This concludes the proof.

The last issue we would like to address in this paper is the following. As the proof of Theorem 5.2 shows, the hardness of $\mu_{G}$ can be deduced from the hardness of $\mu_{G^{\prime}}$, where $G^{\prime}$ is a sparse subgraph of $G$. It is natural to ask whether the hardness of $\mu_{G}$ itself, where $G$ is constructed as in Theorem 3.8, can also be traced to a simple hard subgraph $G^{\prime}$ of $G$. What is the "core" of the hardness? It turns out that $G$ does indeed contain such a subgraph! To avoid technicalities, we bring here only a broad outline of the argument.

First, a purely graph-theoretic argument implies the following.

Proposition 5.3. $G$ contains as a subgraph an expander of a bounded degree (say $\leq 500)$ and size $\Omega(n)$.

Indeed, by the Cheeger Inequality for graphs (cf. [8]), which relates the normalized spectral gap to the normalized edge-expansion, $G$ has edge expansion $\geq d / 4$ where $d$ is the degree of $G$. Choosing each edge of $G$ independently at random with probability $100 / d$, we obtain a graph $\tilde{G}$ which almost has the required properties. Recall that $d$ is large enough $(\approx 100 \log n)$, and hence, by a Chernoff bound, almost surely all sufficiently large subsets of vertices $S$, say $|S| \geq n / 4$, will have an edge-boundary of size at 


\section{NEWMAN, AND Y. RABINOVICH}

least $c_{2} \cdot 100 \cdot|S|$ in $\tilde{G}$, for some constant $c_{2}$ (e.g., $c_{2}=1 / 8$ suffices). Let $D$ be the set of all vertices of degree more than 500. By Chernoff, this set is of size at most $10^{-100} \cdot n$ and has edge boundary of size at most $10^{-50} n$. Thus, in $G^{\prime \prime}=\tilde{G}-D$ all sets $S$ of vertices, of size, say $|S| \geq n / 3$, will have an edge-boundary of size at least $c_{3} \cdot 100 \cdot|S|$, where $c_{3}$ could be taken to be, e. g., $1 / 9$. Next, remove one by one the subsets of vertices $U$ that have fewer than $c_{3} \cdot 100 \cdot|S|$ outgoing edges in the remaining part of the graph. Since the union $W$ of all removed has fewer than $c_{3}|W|$ outgoing edges, we conclude that the size of $W$ is at most $n / 3$. Thus, the graph $G^{\prime}=G^{\prime \prime} \backslash W$ is almost surely a subgraph of $G$ of size $\geq n / 2$, degree $\leq 500$, and edge expansion $\geq c_{3} \cdot 100$. Of course, $G^{\prime}$ is not a Cayley graph anymore, it is not even regular.

Finally, having such a large subgraph $G^{\prime}$ in $G$ implies the hardness of $\mu_{G}$ as asserted by the following proposition.

Proposition 5.4. The existence of such $G^{\prime}$, combined with the property of $G$ (as in Proposition 3.5), that the radius of a $\mu_{G}$-ball of size $n^{\Omega(1)}$ in $G$ is at least $\Omega(\log n)$, implies the hardness of $\mu_{G}$.

Indeed, let $\mu^{\prime}$ denote the restriction of $\mu_{G}$ to $V\left(G^{\prime}\right)$. The hardness of $\mu^{\prime}$ can be proved by employing the Poincare form $F_{G^{\prime}}(\Delta)$ as in Equation (3.1), and using the expansion of $G^{\prime}$ to get, via the Cheeger Inequality for graphs, a lower bound on the first eigenvalue of the Laplacian of $G^{\prime}$.

Now, using the same form $F_{G^{\prime}}(\Delta)$ as in Equation (3.1), this time for $\mu_{G}$, we conclude that the square of the distortion of $\mu_{G}$ is at least $\operatorname{dist}^{2}\left(\mu_{G^{\prime}} \hookrightarrow \ell_{2}\right) \cdot \frac{\operatorname{avg}\left(\mu_{G}^{2}\right)}{\operatorname{avg}\left(\mu_{G^{\prime}}^{2}\right)}$. Since both $\operatorname{avg}\left(\mu_{G}^{2}\right), \operatorname{avg}\left(\mu_{G^{\prime}}^{2}\right) \operatorname{are~} \Theta\left(\log ^{2} n\right)$, the hardness of $\mu_{G}$ follows.

We end with the following open problem concerning hard metrics. In all previous constructions, as well as in the current ones, the metrics that are constructed are hard with respect to NEG and hence with respect to $\ell_{1}$ and $\ell_{2}$. Is there a family of hard metrics that is hard with respect to $\ell_{2}$ but not with respect to NEG ?

\section{References}

[1] N. Alon And Y. Roichman: Random Cayley graphs and expanders. Random Structures Algorithms, 5(2):271-284, 1994. [doi:10.1002/rsa.3240050203]. 128, 129

[2] S. Arora, J. R. Lee, AND A. NaOr: Euclidean distortion and the sparsest cut. In Proc. 37th STOC, pp. 553-562. ACM Press, 2005. [STOC:1060590.1060673]. 126

[3] Y. Aumann And Y. Rabani: An $O(\log k)$ approximate min-cut max-flow theorem and approximation algorithm. SIAM J. Comput., 27(1):291-301, 1998. [doi:10.1137/S0097539794285983]. 126,128

[4] J. Bourgain: On Lipschitz embeddings of finite metric spaces in Hilbert space. Israel J. Math., 52(1-2):46-52, 1985. 125

[5] S. Chawla, A. Gupta, AND H. RÄCKE: Embeddings of negative-type metrics and an improved approximation to generalized sparsest cut. ACM Trans. Algorithms, 4(2):1-18, 2008. [doi:10.1145/1361192.1361199]. 126 
[6] M. M. Deza And M. Laurent: Geometry of Cuts and Metrics. Springer Verlag, 1997. 126

[7] A. Gupta, R. Krauthgamer, And J. R. LeE: Bounded geometries, fractals, and low-distortion embeddings. In Proc. 44th FOCS, pp. 534-543. IEEE Comp. Soc. Press, 2003. 126

[8] S. Hoory, N. Linial, And A. Wigderson: Expander graphs and their applications. Bull. Amer. Math. Soc., 43(4):439-561, 2006. [doi:10.1090/S0273-0979-06-01126-8]. 128, 131

[9] J. JUSTESEN: A class of constructive asymptotically good algebraic codes. IEEE Trans. Inform. Theory, 18(5):652-656, 1972. 130

[10] S. KhOt And A. NAOR: Nonembeddability theorems via Fourier analysis. In Proc. 46th FOCS, pp. 101-112. IEEE Comp. Soc. Press, 2005. [doi:10.1109/SFCS.2005.61]. 126, 130

[11] N. Linial, E. London, AND Y. RABinOVICH: The geometry of graphs and some of its algorithmic applications. Combinatorica, 15(2):215-245, 1995. [doi:10.1007/BF01200757]. 126, 127, 128

[12] F. J. MacWilliams And N. J. A. Sloane: The Theory of Error-Correcting Codes. North Holland, 1977. 130

[13] J. MatoušeK: Lectures on Discrete Geometry. Springer, 2002. 127

[14] S. RAO: Small distortion and volume preserving embeddings for planar and Euclidean metrics. In Proc. 15th Ann. Symp. on Comput. Geometry (SoCG'99), pp. 300-306. ACM Press, 1999. [doi:10.1145/304893.304983]. 126

[15] Avi Wigderson And David XiaO: Derandomizing the Ahlswede-Winter matrix-valued Chernoff bound using pessimistic estimators, and applications. Theory of Computing, 4(1):53-76, 2008. [doi:10.4086/toc.2008.v004a003]. 128

\section{AUTHORS}

Ilan Newman

Department of Computer Science

Haifa University, Haifa, 31905, Israel

ilan@cs.haifa.ac.il

http://cs.haifa.ac.il/ ilan

Yuri Rabinovich

Department of Computer Science

Haifa University, Haifa, 31905, Israel

yuri@cs.haifa.ac.il

http://cs.haifa.ac.il/ yuri 


\section{NEWMAN, AND Y. RABINOVICH}

\section{ABOUT THE AUTHORS}

ILAN NEWMAN has been on the faculty at Haifa University since 1992. He obtained his B. S. and M. S. at the Technion. He received his Ph. D. in 1992 at the Computer Science Department of the Hebrew University under the supervision of Avi Wigderson. The title of his thesis was "On the complexity of simple Boolean functions." His current research interests are in finite metric embeddings, combinatorial algorithms, property testing, and computational complexity.

Born in Odessa, Ukraine, YURI RABINOvich received his Ph. D. in 1993 from the Hebrew University under the supervision of Avi Wigderson and Nati Linial. The title of his thesis was "Theoretical properties of genetic algorithms." Currently he works at the Computer Science Department of Haifa University. He is interested in geometric phenomena in discrete structures in general, and in the theory of finite metric spaces in particular. 